\title{
Sleep apnea syndrome in acromegalic patients: Can morphological evaluation guide us to optimize treatment?
}

\author{
Ana M. Ramos-Levi ${ }^{1} \cdot$ Mónica Marazuela $^{1}$
}

Received: 15 October 2015 / Accepted: 22 October 2015/Published online: 30 October 2015

(C) Springer Science+Business Media New York 2015

Sleep apnea syndrome (SAS) is a common complication in patients with newly diagnosed acromegaly. Different series have noted a relatively high prevalence $(45-80 \%)$, which is significantly higher than that of the general population $(2-4 \%)[1,2]$, although it is probably still under-assessed [3]. In this setting, a recent consensus on acromegaly complications stated that every patient should undergo a careful symptomatic [for example, with an Epworth Sleepiness scale score (ESS)] and/or laboratory assessment for SAS at the time of diagnosis, in collaboration with a respiratory physician [3]. Obstructive SAS (OSAS) is the prevailing form in acromegaly; this occurs due to craniofacial deformations, hypertrophy of pharyngeal soft tissue, macroglossia and thickening of upper airway, all of which hamper the airflow through upper airways. Several predisposing factors can be correlated with the onset of OSAS in acromegaly, such as age, male gender, neck and finger circumference, body mass index, sedatives, alcohol, diabetes mellitus, hypertension, myocardial ischemia, arrhythmias, heart failure, and previous stroke [2]. At the same time, central SAS may develop due to inhibition of the breathing center caused by elevated serum GH/IGF-I levels or to the enhanced somatostatin tone [4]. Therefore, disease-specific parameters, such as high levels of GH/IGF-I and active disease duration, can be also correlated with the severity of SAS [2].

Apart from the gold-standard method polysomnography, there have been techniques, such as cephalometry (which

Mónica Marazuela

monica.marazuela@salud.madrid.org

1 Department of Endocrinology, Hospital Universitario de la Princesa, Instituto de Investigación Princesa, Universidad Autónoma de Madrid, C/Diego de León 62, 28006 Madrid, Spain measures the shape and size of the orofacial skeleton, the size of the velum, and the shape of the upper airways), computed tomography, magnetic resonance imaging (MRI), and nasopharyngoscopy, which have been used to evaluate the existence of anatomical abnormalities of the upper airways and their potential role in the development of OSAS in acromegalic patients [5]. However, their role and potential usefulness in this particular setting have not been fully established. In this issue, Castellani et al. [6] perform a longterm study of SAS in a large cohort of acromegalic patients, and carry out a novel and interesting morphological and functional study with MRI with 3-dimensional elaboration and fiberoptic nasopharyngoscopy (FN) with the Muller maneuver (MM). With this original approach, the authors meticulously assess the occurrence of OSAS in patients with acromegaly. They confirm the high prevalence of SAS in these individuals $(58.6 \%)$ and provide further evidence of the possible anatomical mechanisms involved in the pathophysiology of OSAS. Specifically, their anatomical and dynamic evaluations demonstrate the key role of the length and thickness of the uvula, as it narrows available upper airways and modulates airflow, and they observe how both uvula and tongue sizes are bigger in patients with acromegaly and severe OSAS. But still, they report how residual craniofacial deformations may be as well responsible for an increased apnea/hypopnea index, regardless of soft tissue hypertrophy. In view of these findings, soft tissue involvement and anatomical alterations (either constitutional, or residual) appear to be relevant in the development of this respiratory comorbidity.

Conflicting results have been reported regarding the potential reversibility of SAS after successful treatment of acromegaly. For instance, some studies showed significant improvement after pituitary surgery [7] or somatostatin analogues [8,9], while others observed that SAS persisted 
after recovery of acromegaly in a relatively high percentage of patients, despite a potential association with IGF-I levels and disease duration [1]. In their longitudinal study, Castellani et al. noted how active acromegalic patients who achieved biochemical control, regardless of how they did so (surgery, radiotherapy and/or medical therapy), experienced improvement of their sleep disorder. However, improvement was not as evidently observed in already controlled subjects, in whom OSAS persisted (approximately in $2 / 3$ of patients).

This inconsistency regarding the resolution of SAS despite successful acromegaly treatment advocates for the need to perform a post-treatment evaluation, as it was recommended by the Acromegaly Consensus Group [3], not only in patients with partial or no response to GHlowering therapies, but also after biochemical normalization. When biochemical control is achievable, soft tissue hypertrophy may reverse and OSAS may improve with acromegaly-directed treatment. On the contrary, however, persistence of the respiratory disorder could be due to residual and irreversible skeletal and craniofacial deformations already established. At this time, consultation with maxillofacial surgeons is advised [3], and elective surgery may be necessary. So, once again, we highlight the importance of a prompt evaluation.

It seems that we are underestimating the prevalence and the relevance of SAS, which may account for up to $25 \%$ of causes of death, second only to cardiovascular events [10, 11]. Not only should we assess OSAS at diagnosis of acromegaly, but also during follow-up and at achievement of optimal disease control. This does not necessarily imply the use of sophisticated and refined diagnostic tools such as the ones presented by Castellani et al. What is important is to identify SAS, and this can be done by performing a simple and straightforward test like the ESS. However, if we also evaluate the main physiopathogenic mechanism involved in its development by demonstrating anatomical and functional abnormalities, caused by soft tissue hypertrophy or rather persistent craniofacial deformations, treatment may be targeted towards the predominant alteration. Castellani et al. guide us through the promising usefulness of certain diagnostic tools, and even provide a potential prognostic factor for the expected outcome of treatment of OSAS in acromegalic patients, by differentiating the underlying mechanism of SAS and the "stage" of the disease. With greater sample sizes and long-term follow-up, we will be able to elucidate the role of acromegaly treatment and targeted SAS treatment on the outcome of such a respiratory comorbidity, as well as its potential associated cardio-metabolic conditions. Other influencing factors mentioned above, and issues regarding the relevance of positional changes which could hamper airflow through the upper airways, should also be considered in future studies on this topic.

\section{Compliance with Ethical Standards}

Conflict of interest The authors declare that there is no conflict of interest that could be perceived as prejudicing the impartiality of the research reported.

\section{References}

1. M.V. Davi, L. Dalle Carbonare, A. Giustina, M. Ferrari, A. Frigo, V.L. Cascio, G. Francia, Sleep apnoea syndrome is highly prevalent in acromegaly and only partially reversible after biochemical control of the disease. Eur. J. Endocrinol. 159(5), 533-540 (2008)

2. M.V. Davi, A. Giustina, Sleep apnea in acromegaly: a review on prevalence, pathogenic aspects and treatment. Expert Rev. Endocrinol. Metab. 7, 55-62 (2012)

3. S. Melmed, F.F. Casanueva, A. Klibanski, M.D. Bronstein, P. Chanson, S.W. Lamberts, C.J. Strasburger, J.A. Wass, A. Giustina, A consensus on the diagnosis and treatment of acromegaly complications. Pituitary 16, 294-302 (2013)

4. R.R. Grunstein, K.Y. Ho, C.E. Sullivan, Sleep apnea in acromegaly. Ann. Intern. Med. 115, 527-532 (1991)

5. S. Dostalova, K. Sonka, Z. Smahel, V. Weiss, J. Marek, D. Horinek, Craniofacial abnormalities and their relevance for sleep apnoea syndrome aetiopathogenesis in acromegaly. Eur. J. Endocrinol. 144, 491-497 (2001)

6. C. Castellani, G. Francia, L. Dalle Carbonare, M. Ferrari, E. Viva, R. Cerini, A. Zaccarella, L. Trevisiol, M.V. Davi, Morphological study of upper airways and long-term follow-up of obstructive sleep apnea syndrome in acromegalic patients. Endocrine (2015). doi:10.1007/s12020-015-0659-x

7. L. Sze, C. Schmid, K.E. Bloch, R. Bernays, M. Brändle, Effect of transsphenoidal surgery on sleep apnoea in acromegaly. Eur. J. Endocrinol. 156, 321-329 (2007)

8. M.S. Ip, K.C. Tan, W.C. Peh, K.S. Lam, Effect of Sandostatin LAR on sleep apnoea in acromegaly: correlation with computerized tomographic cephalometry and hormonal activity. Clin Endocrinol (Oxf). 55, 477-483 (2001)

9. G. Tolis, N.G. Angelopoulos, E. Katounda, G. Rombopoulos, V. Kaltzidou, D. Kaltsas, A. Protonotariou, A. Lytras, Medical treatment of acromegaly: comorbidities and their reversibility by somatostatin analogs. Neuroendocrinology 83, 249-257 (2006)

10. L.M. Fatti, M. Scacchi, A.I. Pincelli, E. Lavezzi, F. Cavagnini, Prevalence and pathogenesis of sleep apnea and lung disease in acromegaly. Pituitary 4, 259-262 (2001)

11. A. Colao, D. Ferone, P. Marzullo, G. Lombardi, Systemic complications of acromegaly: epidemiology, pathogenesis, and management. Endocr. Rev. 25, 102-152 (2004) 